\title{
European Genetic Diversity and Susceptibility to Pathogens
}

\author{
Paul J. McLaren ${ }^{\mathrm{a}-\mathrm{c}}$ Jacques Fellay ${ }^{\mathrm{a}-\mathrm{c}}$ Amalio Telenti ${ }^{\mathrm{a}}$

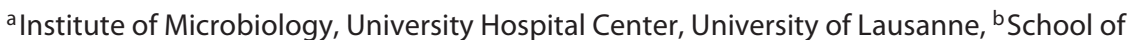 \\ Life Sciences, École Polytechnique Fédérale de Lausanne, and 'Swiss Institute of Bioinformatics, \\ Lausanne, Switzerland
}

\section{Key Words}

Infectious diseases - Immunogenetics · Genome-wide association studies · Exome sequencing · Evolutionary genomics $\cdot$ Host-pathogen interactions

\begin{abstract}
Infectious diseases, both in their endemic and epidemic forms, have shaped the human genome. Ecology has also contributed to geographically constrained pressures on human populations. There are now multiple examples of population-specific genetic variants that modulate susceptibility to infection - several of which have been observed solely in Europeans. The pathogen genome also mutates and adapts to individuals and common alleles in populations. The current understanding has benefited from genomewide association studies as well as from rapid progress in the genetic characterization of Mendelian immunodeficiencies that are defined by susceptibility to specific pathogens. It is expected that current efforts to characterize rare human genetic variants will contribute to the understanding of severe manifestations of common infections in European and other human groups.

(c) 2014 S. Karger AG, Basel
\end{abstract}

\section{Introduction}

Infectious diseases are one of the major driving forces of positive natural selection in human populations, and their effects can be readily observed across the human genome [reviewed in 1]. Due to the ecological context of some infectious diseases, these selected sites are often either population specific or highly differentiated. Perhaps the best-known examples have been described in nonEuropean populations, e.g. the $\mathrm{HbS}$ allele of the beta hemoglobin gene (HBB), which confers resistance to infection by falciparum malaria; similarly, the presence of two copies of a null mutation for the Duffy Antigen Receptor for Chemokines (DARC) confers resistance to infection by Plasmodium vivax. Interestingly, it has been extensively noted that the frequency of both of these alleles is highly increased in malaria-endemic regions [2]. Other resistance alleles, such as the CCR5 $\Delta 32$ mutation conferring protection against HIV-1 infection, have risen to high frequency in European populations, although unlikely due to infection pressure $[3,4]$. Additionally, the evolutionary analysis of inflammatory disease susceptibility loci in European populations has demonstrated evidence of a recent positive selection across these sites and suggested that their currently high frequency may be due to past

Prof. Amalio Telenti

Institute of Microbiology CHUV

CH-1011 Lausanne (Switzerland)

E-Mail amalio.telenti@chuv.ch 
Fig. 1. Chromosomal loci associated with pathogen susceptibility identified in GWAS. TB = Tuberculosis.

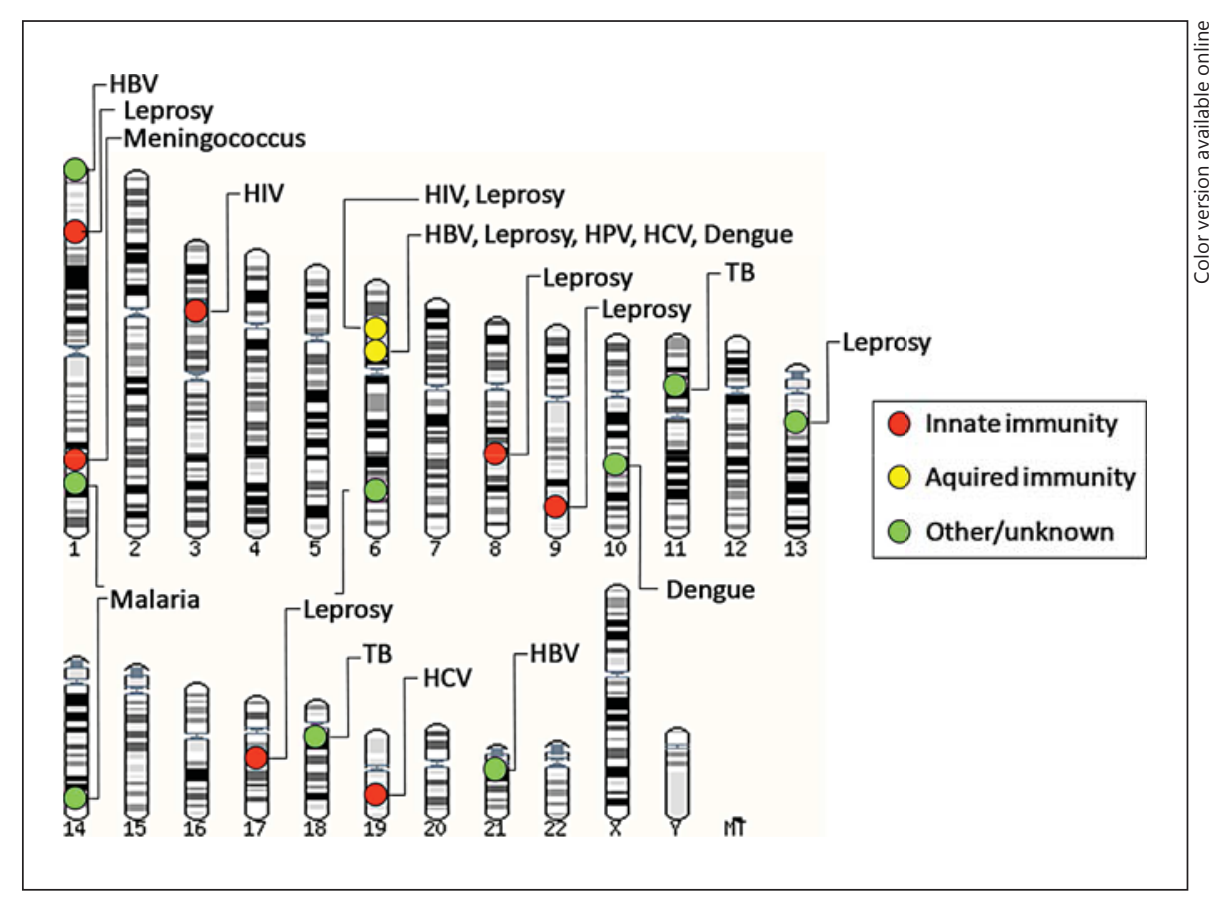

pressure by infectious diseases [5]. In this review, with a particular focus on European populations, we will discuss the impact of common and rare human genetic variation on susceptibility to infection by various pathogens and provide an immunological and evolutionary context for these key variants in human immunity.

\section{Genome-Wide Association Studies in Infectious Diseases}

Similar to other fields of medicine, the speed and accuracy of the identification of genetic loci associated with susceptibility to infectious diseases have followed the emergence of genomics [6]. Since 2007, a number of genome-wide association studies (GWAS) have identified common variants associated with susceptibility to infection, clearance of infection, or progression of disease for several major human pathogens. These variants map prominently to the HLA region, to mediators of innate immunity, and, for about one third of them, to other mechanisms or regions of unknown function (fig. 1; table 1). In general, the trait variance explained by these variants remains at less than $20 \%$ when calculated using an additive genetic model, although in some cases homozygosity may confer a significant degree of protection. Specific to the host-pathogen encounter, part of the un- explained variability may correspond to microbial virulence and epidemiology.

Population-specific differences in the genetic architecture of susceptibility are expected given the geographical constraints and ecology in pathogen distribution as well as the evolutionary pressures from exposure to endemic and, prominently, epidemic outbreaks. This differential architecture is often observable through large differences in allele frequencies at infectious disease susceptibility loci between geographically separated human populations (table 1). Two prominent examples of such population-specific frequency differences are CCR5 32 (rs333) resulting in the deletion of the coreceptor of HIV-1, which is all but absent in non-European populations, and $I L 28 B$ variants that result in differences in the response to $\mathrm{HCV}$.

The CCR5 32 allele is almost exclusive to European populations and has a distinctive north-south gradient, with frequencies ranging from as high as $16 \%$ in some Northern Europeans to less than $4 \%$ in Southern regions (fig. 2) [3]. Homozygosity provides high protection against HIV-1 infection. A possible role of lentiviral (retroviruses including simian and human immunodeficiency viruses) or other pathogen-selective pressures has been suggested on the basis of convergent evolution: multiple primate species present different alleles that result in null function of CCR5 [7]. The null status is not associated 
Table 1. Common variants identified by GWAS as being associated with pathogen susceptibility

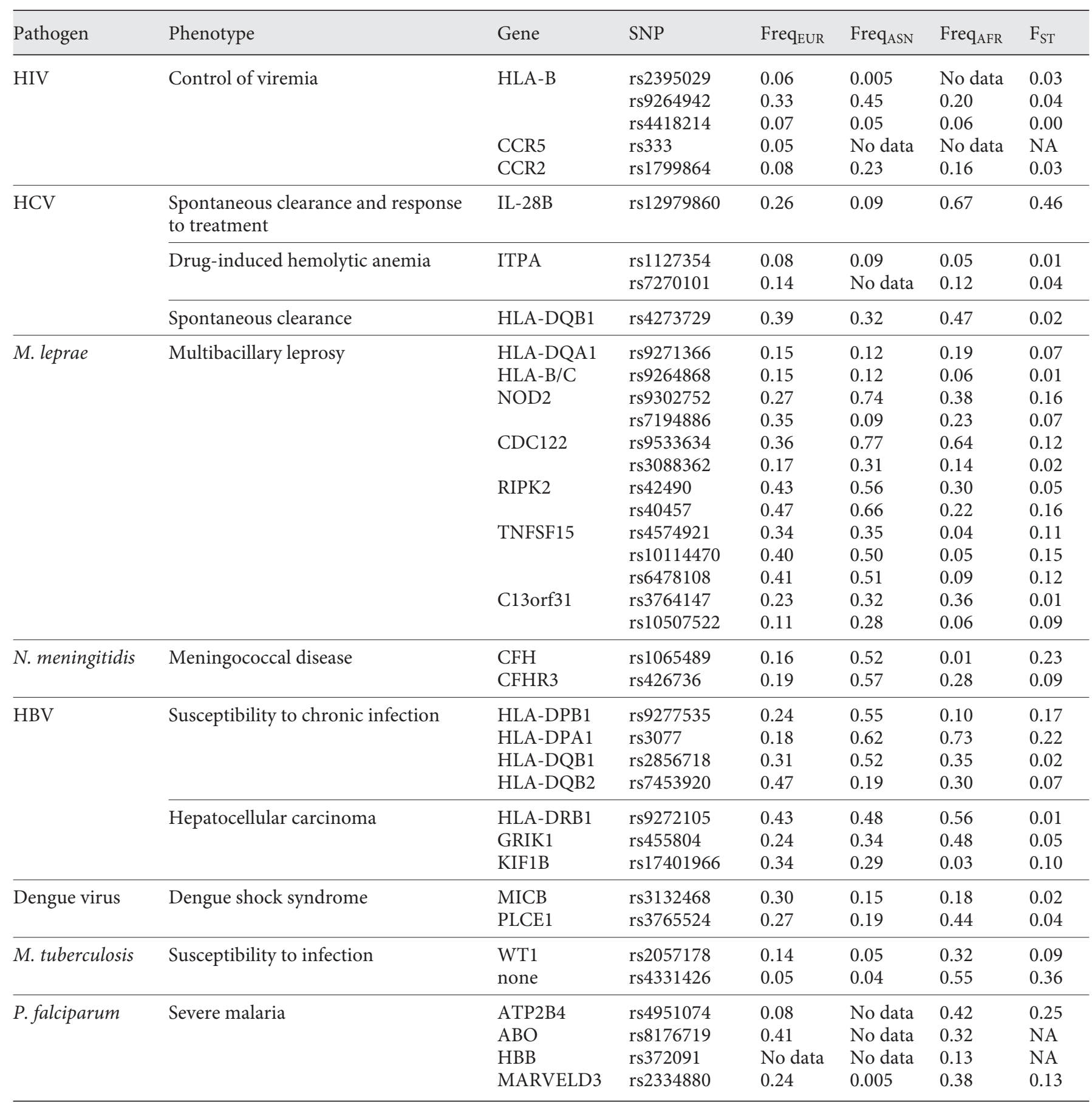

Freq $=$ Frequency $; \mathrm{F}_{\mathrm{ST}}=$ fixation index, where a higher value indicates greater population differentiation. Frequency and $\mathrm{F}_{\mathrm{ST}}$ values are based on European (EUR), Asian (ASN) and African (AFR) 1000 Genomes Project reference populations. F $_{S T}$ values were calculated using the HIERFSTAT package in R (http://www.unil.ch/popgen/softwares/hierfstat.htm). No data indicates that the variant allele is either rare or absent in that population. NA indicates insufficient data to calculate $\mathrm{F}_{\mathrm{ST}}$ values across populations. 


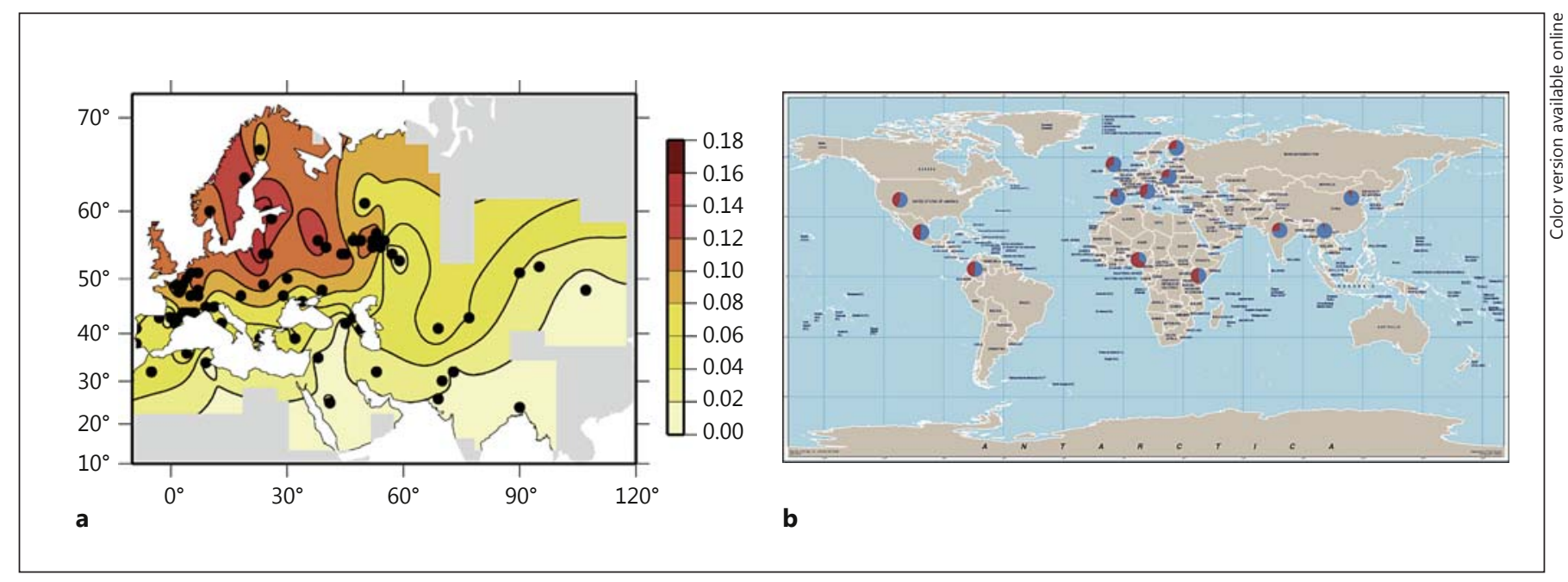

Fig. 2. Population differentiation of protective alleles. a Frequency gradient of CCR5 $\triangle 32$ in Europe. Black dots indicate the sampling locations; from Novembre et al. [3] with permission. b Allele frequency and degree of differentiation of the IL28B protective allele rs12979860(C). The pie charts show the frequency of the C (blue; colors refer to the online version only) and T (red) alleles in each population sampled.

with evidence of immunodeficiency, although there are experimental animal data and human population studies that suggest enhanced susceptibility to a number of pathogens, in particular the West Nile virus [7]. There has been considerable discussion on the selective forces that may have given rise to this allele. The age and origin of CCR5 32 in Europeans remain controversial $[4,8]$.

$I L 28 B$ variants are a good example of population differentiation. There is an Asian-European-African gradient in frequency of the protective allele rs12979860, with the highest frequency observed in Asians (fig. 2). The allele distribution correlates with the rates of spontaneous or treatment-related clearance of HCV infection [9]. The exact role of interferon lamda-3, the product of $I L 28 B$, and the causal role of the different variants, whether in the upstream and promoter regions or the coding region, remain the subject of research [10].

\section{Mendelian Susceptibility to Infection}

Primary immunodeficiencies (PIDs) are rare genetic conditions that affect the development and/or the function of the immune system. They are not only responsible for an increased susceptibility to infection but are also frequently involved in autoimmunity, allergy and malignancy [11]. Linkage analyses and candidate gene studies have been used to explore human susceptibility to severe rare infectious diseases and identify PID-causing genes [12]. Over the past few years, however, advances in sequencing technology have allowed the identification of Mendelian defects related to infectious diseases through exome or genome sequencing, leading to an explosion in the number of newly discovered PIDs. Particularly striking progress has been made in the identification of numerous pathogen-specific immunodeficiencies, which led to the redefinition of the concept of PID $[13,14]$. The first successful application of exome sequencing to a rare infectious disease phenotype was the discovery of a single-gene defect in immunity against HHV-8 in a Turkish child with fatal Kaposi's sarcoma [15]: a splice-site mutation in the STIM1 gene was identified as the disease-causing variant. In another example, a combination of genome-wide linkage analysis and exome sequencing was used to demonstrate that gain-of-function mutations of STAT1 are responsible for chronic mucocutaneous candidiasis disease [16].

Today, rare variants conferring extreme susceptibility to specific infectious agents are identified at an ever-increasing rate [11]. Important in this context is the observation reported by the 1000 Genomes Project that the majority of variants with a low minor allele frequency (MAF $<0.5 \%)$ are restricted to a single population [17]. This is explained by a strong excess of rare variants in human populations, attributable to the recent explosive demographic growth [18-21]. Therefore, deleterious alleles are 
likely to be population specific, and the newly discovered PIDs will often be observed in restricted geographical areas. The European Society for Immunodeficiencies (ESID; http://www.esid.org) is running a pan-European registry for PIDs (the ESID database), in which about 15,000 patients have been registered to date [22]. Based on the reported data, the global prevalence of PIDs in European countries is estimated to be at least 5 per 100,000 inhabitants. Marked differences are observed in the specific type of PIDs reported between countries and populations, which can be partially explained by demographic history (e.g. isolated vs. mixed population, founder effects and migration rates) and by the genetic mechanism involved (e.g. recessive deleterious alleles and de novo dominant mutations). Of note, even if PIDs are by definition genetic diseases, the actual defect is still unknown in many patients: a genetic cause has been identified in fewer than half of the patients registered in the ESID database. Overall, about $10 \%$ of cases are linked with a consanguineous background and $20 \%$ are described as familial cases (i.e. other members of the family also presented with a PID).

Depending on the human genetic underpinning of common infectious diseases, PIDs could also be responsible for the tail of their phenotypic distribution. More specifically, individuals with inborn innate deficiencies are likely to be overrepresented among patients with the most dramatic clinical presentation of common infections. For example, recent GWAS and family studies strongly suggest that the human genetics of tuberculosis involves a continuous spectrum from Mendelian to complex predisposition. Patients with IL-12R $\beta 1$ deficiency have been shown to suffer from severe forms of the disease, yet they were not distinguishable from the rest of the patient population based on clinical findings alone [23].

\section{Immunogenetics - Human Leukocyte Antigen}

The major histocompatibility complex (MHC) locus on chromosome 6 (spanning from approximately 25.6 to $33.2 \mathrm{Mb}$ for the extended MHC) includes the human leukocyte antigen (HLA) genes. Owing to their function in adaptive immunity, HLA class I and class II genes have shown particular importance to infection susceptibility. This region carries one of the broadest and most complex patterns of linkage disequilibrium genome-wide and contains more than 400 genes, many with clear relevance to innate and adaptive immunity [24]. This high density of genes and the complex correlation structure complicate the in-depth understanding of genetic associations in this region [25]. These genes are incredibly diverse, with thousands of reported allelic variants differing in both their nucleotide and amino acid sequences. For some infections, different alleles confer a range of effect sizes on infectious disease susceptibility (from highly protective to highly harmful) and vary greatly in frequency between populations.

As seen in figure 1, the vast majority of GWAS performed on infectious disease susceptibility phenotypes have identified strong associations in the HLA region, including susceptibility to dengue shock syndrome [26], acquisition of Mycobacterium leprae [27], HPV seropositivity [28], HBV acquisition [29], HBV-induced hepatocellular carcinoma [30, 31], spontaneous clearance of $\mathrm{HCV}$ infection [32, 33] and HIV control [34, 35]. Furthermore, with the exception of the study on dengue shock syndrome, all of these studies either directly or indirectly implicate allelic diversity in HLA class I (HIV) or class II genes as the major driving force behind the observed SNP associations. In line with GWAS on inflammatory diseases, associations in the HLA region have comparatively large odds ratios (and thus explain more of the trait variance) compared to non-HLA hits, underscoring the large biological impact of variation in this region.

The role of HLA class I allelic diversity (particularly in $H L A-B$ ) in modifying HIV-1 disease outcome has been well described. Placing this in the genome-wide context, the first GWAS of an infectious disease phenotype (HIV-1 viral load) observed that the top statistical signal rs2395029 is a near perfect proxy in European populations for the strongly protective classical allele HLA-B*57:01 [34]. Adding further biological insight into this, an in silico fine-mapping effort demonstrated that the majority of the SNP and classical allele association signal could be explained by a small number of amino acid residues in the binding pocket of HLA-B [35].

Owing to geographical differences in historical infection pressure, the frequency of SNPs, classical alleles and their component amino acids varies across populations [36]. For instance, HLA-B*57:01, albeit common in Europeans, is not present in populations of African ancestry, although the closely related molecule $B^{*} 57: 03$ also slows disease progression in Africans and African-Americans $[37,38]$. Though the strong role of HLA variation in influencing infectious disease susceptibility is incontrovertible, it remains to be seen whether variation in other adaptive immune molecules will play a similar role. Larger sample sizes for GWAS and/or genome-sequencing studies will be necessary to further address this issue. 
Unique to the host-pathogen analysis is the possibility to apply genome-to-genome strategies to highlight sites of genomic conflict. In a recent study, we used paired human and HIV sequence data from 1,071 individuals to run $>3,000$ genome-wide scans [39], testing for associations between host DNA polymorphisms and HIV sequence variation, while considering human and viral population structures. There were significant human SNP associations with a total of $48 \mathrm{HIV}-1$ amino acid variants of the virus. All associations mapped to the HLA class I region. A similar strategy can be used to study other infectious diseases (e.g. infections by $\mathrm{HBV}, \mathrm{HCV}$, herpes viruses or mycobacterium tuberculosis) as long as polymorphic host factors exert sufficient selective pressure to trigger escape mutations in the pathogen.

\section{Innate Immunity}

Several common and very rare variants in innate immune genes have been associated with susceptibility to infectious diseases. It is also well accepted that genes and cellular pathways enriched for signals of positive selective pressure are frequently involved in the immune response $[40,41]$. The underlying concept is that evasion from and co-evolution with pathogens are among the strongest evolutionary pressures, resulting in signals identifiable through comparative genomics. The signals of positive selection are most pronounced at domains of direct interaction with a pathogen [42]. The HIV restriction factors TRIM5 $\alpha$, APOBEC3G, BST2 and SAMHD1 are relevant examples of genes that have undergone positive selection [42].

There is also the expectation that genes of the innate immune system may carry rare variants that determine differences in susceptibility to infection across populations and individuals. The consequences of carrying a functional or null mutation of a gene, as has been identified for many Mendelian disorders, may be pathogen specific and thus only expressed upon an eventual hostpathogen encounter. A significant challenge to progress in the field is the ability to identify the perimeter of the innate immunity and specifically to identify characteristics of genes that can be prioritized for in-depth genetic and functional analyses [43]. Some basic metrics that include evidence of positive selection, gene duplication and enrichment for nonsynonymous variants can be applied to short-list genes of innate immunity - in particular the set of genes that are increasingly described as 'cell-autonomous immunity', - i.e. those genes that participate in intracellular defense against invading pathogens, as opposed to soluble factors such as cytokines. One of the benefits of short-list innate immunity genes is their ability to enhance the precision of analyses of rare human variation, in particular for the study of the more severe manifestations of common infectious disorders.

\section{Conclusions}

Lessons from GWAS and the study of Mendelian disorders underscore the relevance of population history and positive selection pressures from diseases that are often ecologically restricted. Studies of pathogen genomes complete the picture by demonstrating the properties of adaptation to the population as a result of the allele frequency-dependent selection [44]. In the near future, we might be able to interpret rare human variation in the context of selective susceptibility to pathogens.

We have now entered an era in which human genetic variation can be systematically characterized, including at the level of very rare variants. Recent studies demonstrated that exome sequencing in a very small number of individuals is an efficient strategy for investigating the mechanisms underlying the pathogenesis of severe infectious diseases (see above). A crucial advantage of highthroughput sequencing technology versus genotyping methods is the possibility of identifying very rare mutations with deleterious effects, which are kept at very low frequency by evolutionary pressure and are often population specific. Large genomic and phenotypic data sets are needed to catalog all human genetic variants and correlate them with potential clinical consequences. The current convergence of biobanking, electronic health records and clinical genomics offers an unprecedented opportunity to accelerate the discovery of deleterious mutations and to further elucidate the genetic basis of susceptibility to infection.

\section{Acknowledgement}

This work is supported by the Swiss National Foundation (CRSII3_147665/1). 


\section{References}

1 Barreiro LB, Quintana-Murci L: From evolutionary genetics to human immunology: how selection shapes host defence genes. Nat Rev Genet 2010;11:17-30.

$>2$ Allison AC: Protection afforded by sickle-cell trait against subtertian malareal infection. $\mathrm{Br}$ Med J 1954;1:290-294.

3 Novembre J, Galvani AP, Slatkin M: The geographic spread of the CCR5 Delta32 HIV-resistance allele. PLoS Biol 2005;3:e339.

$\checkmark 4$ Hedrick PW, Verrelli BC: 'Ground truth' for selection on CCR5-Delta32. Trends Genet 2006;22:293-296.

5 Raj T, Kuchroo M, Replogle JM, Raychaudhuri S, Stranger BE, et al: Common risk alleles for inflammatory diseases are targets of recent positive selection. Am J Hum Genet 2013;92: 517-529.

$\checkmark 6$ de Bakker PI, Telenti A: Infectious diseases not immune to genome-wide association. Nat Genet 2010;42:731-732.

$>7$ Telenti A, Egger M: Identifying safety concerns from genetic data: lessons from the development of CCR5 inhibitors. Antivir Ther 2007;12:147-148.

8 Sabeti PC, Walsh E, Schaffner SF, Varilly P, Fry B, et al: The case for selection at CCR5Delta32. PLoS Biol 2005;3:e378.

$>9$ Thomas DL, Thio CL, Martin MP, Qi Y, Ge $\mathrm{D}$, et al: Genetic variation in IL28B and spontaneous clearance of hepatitis $\mathrm{C}$ virus. Nature 2009;461:798-801.

$>10$ di Iulio J, Ciuffi A, Fitzmaurice K, Kelleher D, Rotger M, et al: Estimating the net contribution of interleukin-28B variation to spontaneous hepatitis $\mathrm{C}$ virus clearance. Hepatology 2011;53:1446-1454.

-11 Al-Herz W, Bousfiha A, Casanova JL, Chapel $\mathrm{H}$, Conley ME, et al: Primary immunodeficiency diseases: an update on the classification from the international union of immunological societies expert committee for primary immunodeficiency. Front Immunol 2011;2:54.

12 Casanova JL, Abel L: Inborn errors of immunity to infection: the rule rather than the exception. J Exp Med 2005;202:197-201.

-13 Alcais A, Quintana-Murci L, Thaler DS, Schurr E, Abel L, et al: Life-threatening infectious diseases of childhood: single-gene inborn errors of immunity? Ann NY Acad Sci 2010;1214:18-33.

14 Hill AV: Evolution, revolution and heresy in the genetics of infectious disease susceptibility. Philos Trans R Soc Lond B Biol Sci 2012; 367:840-849.

-15 Byun M, Abhyankar A, Lelarge V, Plancoulaine S, Palanduz A, et al: Whole-exome sequencing-based discovery of STIM1 deficiency in a child with fatal classic Kaposi sarcoma. J Exp Med 2010;207:2307-2312.

-16 Liu L, Okada S, Kong XF, Kreins AY, Cypowyj S, et al: Gain-of-function human STAT1 mutations impair IL-17 immunity and underlie chronic mucocutaneous candidiasis. J Exp Med 2011;208:1635-1648.
17 Genomes Project C, Abecasis GR, Auton A, Brooks LD, DePristo MA, et al: An integrated map of genetic variation from 1,092 human genomes. Nature 2012;491:56-65.

18 Coventry A, Bull-Otterson LM, Liu X, Clark AG, Maxwell TJ, et al: Deep resequencing reveals excess rare recent variants consistent with explosive population growth. Nat Commun 2010;1:131.

19 Nelson MR, Wegmann D, Ehm MG, Kessner D, St Jean P, et al: An abundance of rare functional variants in 202 drug target genes sequenced in 14,002 people. Science 2012;337: $100-104$.

20 Tennessen JA, Bigham AW, O’Connor TD, $\mathrm{Fu}$ W, Kenny EE, et al: Evolution and functional impact of rare coding variation from deep sequencing of human exomes. Science 2012;337:64-69.

$21 \mathrm{Fu}$ W, O'Connor TD, Jun G, Kang HM, Abecasis $\mathrm{G}$, et al: Analysis of 6,515 exomes reveals the recent origin of most human protein-coding variants. Nature 2013;493:216-220.

22 Gathmann B, Binder N, Ehl S, Kindle G, Party ERW: The European internet-based patient and research database for primary immunodeficiencies: update 2011. Clin Exp Immunol 2012;167:479-491.

23 Rezaei N, Aghamohammadi A, Mansouri D, Parvaneh N, Casanova JL: Tuberculosis: a new look at an old disease. Expert Rev Clin Immunol 2011;7:129-131.

24 Horton R, Wilming L, Rand V, Lovering RC, Bruford EA, et al: Gene map of the extended human MHC. Nat Rev Genet 2004;5:889899.

25 de Bakker PI, Raychaudhuri S: Interrogating the major histocompatibility complex with high-throughput genomics. Hum Mol Genet 2012;21:R29-R36.

26 Khor CC, Chau TN, Pang J, Davila S, Long HT, et al: Genome-wide association study identifies susceptibility loci for dengue shock syndrome at MICB and PLCE1. Nat Genet 2011;43:1139-1141.

-27 Zhang FR, Huang W, Chen SM, Sun LD, Liu $\mathrm{H}$, et al: Genomewide association study of leprosy. N Engl J Med 2009;361:2609-2618.

28 Chen D, McKay JD, Clifford G, Gaborieau V, Chabrier A, et al: Genome-wide association study of HPV seropositivity. Hum Mol Genet 2011;20:4714-4723.

29 Mbarek H, Ochi H, Urabe Y, Kumar V, Kubo $\mathrm{M}$, et al: A genome-wide association study of chronic hepatitis B identified novel risk locus in a Japanese population. Hum Mol Genet 2011;20:3884-3892.

30 Jiang DK, Sun J, Cao G, Liu Y, Lin D, et al: Genetic variants in STAT4 and HLA-DQ genes confer risk of hepatitis B virus-related hepatocellular carcinoma. Nat Genet 2013;45:72-75.
31 Li S, Qian J, Yang Y, Zhao W, Dai J, et al: GWAS identifies novel susceptibility loci on 6p21.32 and 21q21.3 for hepatocellular carcinoma in chronic hepatitis B virus carriers. PLoS Genet 2012;8:e1002791.

32 Rauch A, Kutalik Z, Descombes P, Cai T, Di Iulio J, et al: Genetic variation in IL28B is associated with chronic hepatitis $\mathrm{C}$ and treatment failure: a genome-wide association study. Gastroenterology 2010;138:1338$1345,1345 \mathrm{e} 1-\mathrm{e} 7$.

33 Duggal P, Thio CL, Wojcik GL, Goedert JJ, Mangia A, et al: Genome-wide association study of spontaneous resolution of hepatitis $\mathrm{C}$ virus infection: data from multiple cohorts. Ann Intern Med 2013;158:235-245.

-34 Fellay J, Shianna KV, Ge D, Colombo S, Ledergerber B, et al: A whole-genome association study of major determinants for host control of HIV-1. Science 2007;317:944-947.

35 Pereyra F, Jia X, McLaren PJ, Telenti A, de Bakker PI, et al: The major genetic determinants of HIV-1 control affect HLA class I peptide presentation. Science 2010;330:15511557.

36 Prugnolle F, Manica A, Charpentier M, Guegan JF, Guernier V, et al: Pathogen-driven selection and worldwide HLA class I diversity. Curr Biol 2005;15:1022-1027.

37 Kiepiela P, Leslie AJ, Honeyborne I, Ramduth D, Thobakgale C, et al: Dominant influence of HLA-B in mediating the potential co-evolution of HIV and HLA. Nature 2004;432:769775 .

38 McLaren PJ, Ripke S, Pelak K, Weintrob AC, Patsopoulos NA, et al: Fine-mapping classical HLA variation associated with durable host control of HIV-1 infection in African Americans. Hum Mol Genet 2012;21:4334-4347.

39 Bartha I, Carlson J, Brumme C, McLaren P, Brumme Z, et al: A genome-to-genome analysis of associations between human genetic variation, HIV-1 sequence diversity, and viral control. Elife 2013;2:e01123.

40 Kosiol C, Vinar T, da Fonseca RR, Hubisz MJ Bustamante CD, et al: Patterns of positive selection in six Mammalian genomes. PLoS Genet 2008;4:e1000144.

41 Daub JT, Hofer T, Cutivet E, Dupanloup I, Quintana-Murci L, et al: Evidence for polygenic adaptation to pathogens in the human genome. Mol Biol Evol 2013;30:1544-1558.

42 Daugherty MD, Malik HS: Rules of engagement: molecular insights from host-virus arms races. Annu Rev Genet 2012;46:677700 .

43 Rausell A, McLaren PJ, Telenti A: HIV and innate immunity - a genomics perspective. F1000Prime Rep 2013;5:29.

44 Telenti A, Beckmann JS, Mallal S: HLA and HIV: modeling adaptation to moving targets. Pharmacogenomics J 2003;3:254-256. 